\title{
Evaluation of bone healing using rhBMP-2 soaked hydroxyapatite in ridge augmentation : Prospective case observational study
}

Kim Sang Yun ${ }^{1 *}$, Kim Young-Kyun ${ }^{1,2}$

${ }^{1}$ Department of Oral and Maxillofacial Surgery, Section of Dentistry, Seoul National University Bundang Hospital, Seongnam , Korea

${ }^{2}$ Department of Dentistry \& Dental Research Institute, School of Dentistry, Seoul National University, Seoul, Korea

\section{Introduction}

NOVOSIS®-Dent (CG Bio, Seongnam, Korea) is a hydroxyapatite-based alloplastic bony substitute containing recombinant human bone morphogenetic protein-2 (RhBMP-2). RhBMP-2 is used as a multi-pore ceramic supporter to promote bone regeneration when used in combination with synthetic grafting materials

\section{Purpose}

We performed a prospective, cohort study to evaluate the effectiveness of alveolar ridge augmentation using rhBMP-2 soaked hydroxyapatite and to describe complications associated with this procedure.

\section{Materials and Methods}

\section{Subjects :}

We enrolled 10 dental implant candidates in our study : four men and six women (average age : $58.5+8.6$ years)

They were divided into experimental and control groups.

\section{Methods :}

In preparation for the implants, alveolar ridge augmentation was performed in several edentulous areas, including the posterior maxilla as well as the anterior and posterior mandible.

the control group $(n=5)$

: Bio-Oss ${ }^{\circledR}$ (Geistlich Biomaterials, Swiss) bone graft material was used

the experimental group $(n=5)$

: NOVOSIS®-Dent was used

Cone beam computed tomography (CBCT) was taken immediately after the procedure and again four months later to evaluate relative changes in bone volume and resorption rate of the graft material.

Eight patients received dental implants in Seoul National University Bundang Hospital, while the others received implants in local clinics.

\section{Results}

1) No postoperative complications were found.

\section{2) CBCT radiographs}

: alveolar bone widths (experimental: $1.75 \pm 0.85 \mathrm{~mm}$, control: $2.52 \pm 0.18 \mathrm{~mm}$ ) and alveolar bone heights (experimental: $1.57 \pm 0.28 \mathrm{~mm}$, control: $1.68 \pm 0.17 \mathrm{~mm}$ ) were increased in both groups.

(1) Resorption rates in the alveolar bone widths : experimental group : $31.49+7.42 \%$ control group : $29.73+8.79 \%$

(1) Resorption rates in the alveolar bone heights : experimental group : $52.58 \pm 6.46 \%$ control group : $39.17 \pm 21.80 \%$

\section{3) histologic examination}

: there was an increase in new bone formation surrounding the graft material.

The relative new bone formation ratios

: experimental group : $35.18 \pm 19.68 \%$ control group : $28.89 \pm 10.25 \%$

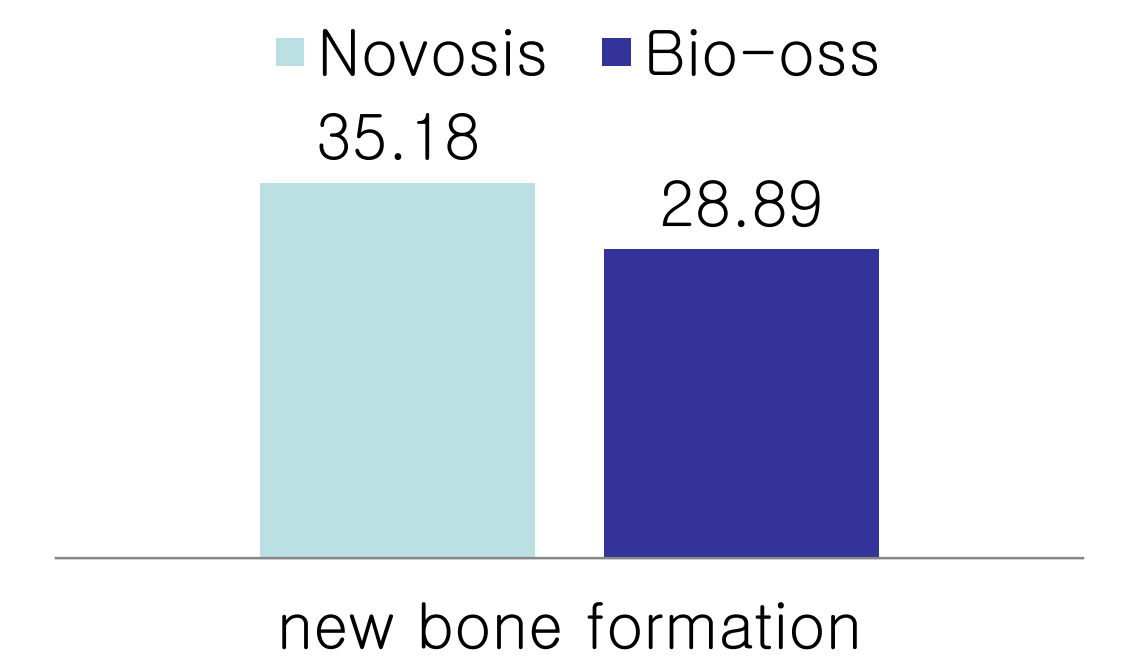

\section{Conclusions}

We found that ridge augmentation with NOVOSIS®Dent is beneficial prior to dental implantation and does not seem to be associated with any serious postoperative complications. 